\title{
Recording local field potential and neuronal activity with tetrodes in epileptic patients
}

Elodie Despouy a,b,c, Jonathan Curot ${ }^{\mathrm{a}, \mathrm{b}, \mathrm{d}}$, Leila Reddy ${ }^{\mathrm{a}, \mathrm{b}}$, Lionel G. Nowak ${ }^{\mathrm{a}, \mathrm{b}}$, Martin Deudon ${ }^{\mathrm{a}, \mathrm{b}}$, Jean-Christophe Sol ${ }^{e, f}$, Jean-Albert Lotterie ${ }^{\mathrm{e}, \mathrm{g}}$, Marie Denuelle ${ }^{\mathrm{d}}$, Ali Maziz ${ }^{\mathrm{h}}$, Christian Bergaud $^{\mathrm{h}}$, Simon J. Thorpe ${ }^{\mathrm{a}, \mathrm{b}}$, Luc Valton ${ }^{\mathrm{a}, \mathrm{b}, \mathrm{d}}$, Emmanuel J. Barbeau ${ }^{\mathrm{a}, \mathrm{b}}$

a- Centre de Recherche Cerveau et Cognition, Université de Toulouse, Université Paul Sabatier Toulouse, Toulouse, F-31330, France

b- Centre National de la Recherche Scientifique CerCo, Toulouse, F-31052, France

c- DIXI Medical, Chaudefontaine, F-25640 France

d- Explorations neurophysiologiques, Hôpital Purpan, Université de Toulouse, Toulouse, F31300, France

e- INSERM, U1214, TONIC, Toulouse Mind and Brain Institute, Toulouse, F-31052, France

f- Neurochirurgie, Hôpital Purpan, Université de Toulouse, Toulouse, F-31300, France

g- Radiochirurgie stéréotaxique, Hôpital Purpan, Université de Toulouse, Toulouse, F-31300, France

h- LAAS-CNRS, Université de Toulouse, CNRS, Toulouse, F-31400, France.

Corresponding author: Elodie Despouy, elodie.despouy@cnrs.fr 


\section{ABSTRACT}

Background: Recordings with tetrodes have proven to be more effective in isolating single neuron spiking activity than with single microwires. However, tetrodes have never been used in humans. We report on the characteristics, safety, compatibility with clinical intracranial recordings in epileptic patients, and performance, of a new type of hybrid electrode equipped with tetrodes.

New Method: 240 standard clinical macroelectrodes and 102 hybrid electrodes were implanted in 28 patients. Hybrids (diameter $800 \mu \mathrm{m}$ ) are made of 6 or 9 macro-contacts and 2 or 3 tetrodes (diameter $70-80 \mu \mathrm{m}$ ).

Results: No clinical complication or adverse event was associated with the hybrids. Impedance and noise of recordings were stable over time. The design enabled multiscale spatial analyses that revealed physiopathological events which were sometimes specific to one tetrode, but could not be recorded on the macro-contacts. After spike sorting, the single-unit yield was similar to other hybrid electrodes and was sometimes as high as $>10$ neurons per tetrode.

Comparison with Existing Method(s): This new hybrid electrode has a smaller diameter than other available hybrid electrodes. It provides novel spatial information due to the configuration of the tetrodes. The single-unit yield appears promising.

Conclusions: This new hybrid electrode is safe, easy to use, and works satisfactorily for conducting multi-scale seizure and physiological analyses. 


\section{HIGHLIGHTS}

- First in vivo tetrode recordings in humans

- No clinical complications or adverse events

- On average $2.08 \pm 2.86$ single units per tetrode (sometimes $>10$ )

\section{KEYWORDS}

Hybrid electrode; depth electrode; tetrode; intracranial EEG; single unit; epilepsy

\section{ABBREVIATIONS}

ACSF: artificial cerebrospinal fluid; EIS: electrochemical impedance spectroscopy; MRI: magnetic resonance imaging; RMS: root mean square; SEEG: stereo-electroencephalography 


\section{INTRODUCTION}

About $30 \%$ to $40 \%$ of epileptic patients suffer from drug refractory epilepsy (Kwan et al., 2011; Laxer et al., 2014; Dalic and Cook, 2016). Epilepsy surgery in these patients provides a good rate of seizure control, with $58-73 \%$ of patients seizure-free two years after surgery (Capraz et al., 2015). Implantation of subdural and/or depth electrodes is often required prior to epilepsy surgery to delineate the seizure onset zone (Behrens et al., 1994; Yang et al., 2014; Podkorytova et al., 2016; Isnard et al., 2018).

Hybrid depth electrodes that combine both macro-contacts and microwires have been used in the investigation of drug-refractory epilepsy for approximately 45 years (Babb et al., 1973; Fried et al., 1999), although this has only been in a few centers worldwide. They provide a unique opportunity to study neuronal activity in the human brain for a better understanding of the mechanisms of epileptogenesis (Keller et al., 2010; Alvarado-Rojas et al., 2013; Lambrecq et al., 2017; Schevon et al., 2019), seizure propagation (Eissa et al., 2017) and seizure prediction (Bower et al., 2008; Park et al., 2014; Misra et al., 2017), and of the neurobiological basis of cognition (Fried et al., 1997; Kreiman et al., 2002; Quiroga et al., 2005; Mormann et al., 2008; Rutishauser et al., 2010; Reddy et al., 2015; Staresina et al., 2019). They are also more effective in detecting pathological activities such as high-frequency oscillations (Zijlmans et al., 2017; Despouy et al., 2019) and microseizures (Schevon et al, 2008; Stead et al., 2010). They are as safe and effective as standard clinical macroelectrodes for intracranial monitoring (Hefft et al., 2013; Carlson et al., 2018).

Since the end of the 1990s, the most commonly used hybrid electrode has been a bundle of microwires (up to 9, each $40 \mu \mathrm{m}$ in diameter) inserted inside the shaft of the electrode and which exit at the tip of the macroelectrode (Babb et al., 1973; Fried et al., 1999). Despite their 
success, such hybrid electrodes suffer from several drawbacks: 1- the diameter of the macroelectrode $(1,300 \mu \mathrm{m})$ is large compared to other standard clinical macroelectrodes used for intracranial monitoring $(800 \mu \mathrm{m}) .2$ - there is an imperfect spatial correspondence between the macrocontact and microelectrode recording sites due to the extension of the microwires by approximately $5 \mathrm{~mm}$ off the tip of the macroelectrode, i.e. macro- and microelectrodes may not record the same neuronal populations; 3- the length of this extension limits the number of brain regions in which these hybrid electrodes can be implanted; 4- It is difficult to localize the microelectrodes as they spread out of the shaft like an umbrella, i.e. the spatial relationship between the different microwires is unknown which might present a difficulty during spike sorting.

The use of tetrodes in animal studies has led to a major improvement in single-unit isolation (Buzsáki, 2004). There are two independent challenges: (1) some areas have high cell densities in which cell bodies are closely packed together and cells can fire in a complex spike pattern; (2) during burst firing, the height of the action potential can vary in amplitude by as much as 50\% (Kandel and Spencer, 1961; Ranck, 1973). The use of a stereotrode (two microwires twisted together) improves the detection of single units compared to single wire recording: cells with different ratios of distances from the two electrode tips have different spike amplitude ratios when recorded on two channels (McNaughton et al., 1983). According to the same logic, tetrode configurations (four microwires bundled together) have been shown to further improve the yield of single units at single recording sites (O'Keefe and Recce, 1993; Wilson and McNaughton, 1993; Gray et al. 1995; Buzsáki 2004). Therefore, up to nine neurons at a time could be recorded from a single tetrode (Gray et al., 1995), although in principle, many more could be recorded (Henze et al., 2000). Tetrode configuration has been 
highly valuable to record the neuronal dynamics of interneurons and principal cells during ictogenesis in in vitro analyses of animal brain slices (Lévesque et al., 2016, 2018).

In this study, we describe a new hybrid electrode which has been under clinical investigation in our epilepsy center since 2015. It contains two or three tetrodes inserted in a macroelectrode of the same diameter as standard clinical macroelectrodes (800 $\mu \mathrm{m})$. Once the hybrid electrode is inserted in the brain, the tetrodes can be extended up to $2 \mathrm{~mm}$ with a micrometer screw. Because the tetrodes extend between two macrocontacts, they allow a true multiscale recording of the same brain region. To our knowledge, this is the first time that tetrodes have been used in a clinical trial in humans. Therefore, the effectiveness in humans was unknown. The purpose of this study was to report on the characteristics of this new hybrid electrode, its safety and its ability to record EEG signals and neuronal activity at several spatial scales simultaneously. Our results suggest that tetrodes may be as useful in humans as in animal studies, both from a clinical and an electrophysiological perspective.

\section{METHODS}

\subsection{Patients}

The patients included in this study suffered from drug-resistant epilepsy. They were included between 2015 and 2018. They had a detailed pre-surgical evaluation including medical history and neurological examination, neuropsychological testing, cerebral MRI, and long-term video-EEG monitoring. All patients had functional imagery such as 18-FDG positron emission tomography and some patients had ictal single photon emission computer tomography. Due to the fact that noninvasive assessments failed to localize the seizure onset zone with certainty, patients underwent stereo-electroencephalography (SEEG) on the 
Toulouse epilepsy unit so that the seizure onset zone could be more precisely delineated. SEEG was performed as part of the patients' regular clinical care. Intracranial EEG electrodes were implanted stereotaxically according to Talairach's method (Talairach et al. 1992). Each patient received detailed information concerning the objectives of the SEEG technique before intracerebral electrode implantation. They were also given the details and informed about the use of these new hybrid electrodes, after which they agreed to participate in the study (ClinicalTrials Identifier: NCT02491476). Implantation of the hybrid electrodes and the use of the data were approved by the local ethics committee and the French Drug and Health Product Safety Agency (CPP Sud-Ouest et Outre-Mer I, no.1-14-23 and ANSM 2014-A00747-40).

\subsection{Electrodes}

Up to 12 semi-rigid multi-contact standard clinical depth macroelectrodes and up to 4 new hybrid electrodes were implanted in each patient. The macroelectrodes (Microdeep, DIXI Medical, France) were $0.8 \mathrm{~mm}$ in diameter and contained 5-18 contacts (platinum/iridium). The contacts were $2 \mathrm{~mm}$ long and were regularly spaced $1.5 \mathrm{~mm}$ apart.

The hybrid electrodes (DIXI Medical, France) consisted of a standard macroelectrode (diameter: $0.8 \mathrm{~mm}$ ) equipped with two or three tetrodes that protruded up to $2 \mathrm{~mm}$ from the shaft between the first and second most distal macrocontacts (Fig. 1). A micrometer screw was used to extend the Tetrodes from the electrode shaft after implantation. The tetrodes protruded from the shaft at a $30^{\circ}$ angle. Each tetrode was made of 4 tungsten wires, $20 \mu \mathrm{m}$ in diameter. The theoretical surface of each microcontact was $6.28 \times 10^{-4} \mathrm{~mm}^{2}$. Each tetrode was 70-80 $\mu \mathrm{m}$ in diameter. For hybrid electrodes equipped with two tetrodes, tetrodes were at a $180^{\circ}$ angle in relation to each other. They also had nine $2 \mathrm{~mm}$-long macrocontacts regularly 
spaced at intervals along the shaft according to the overall length of the electrode. Three models with 2 tetrodes were available in a total exploration length / space between contacts (in $\mathrm{mm}$ ) of: $33.2 / 1.9 ; 40.4 / 2.8 ; 50.8 / 4.1$. The length of the electrodes was chosen according to the depth of the cerebral target. For hybrid electrodes equipped with three tetrodes, the tetrodes were at a $120^{\circ}$ angle in relation to each other. They had six $2 \mathrm{~mm}$-long macrocontacts, three distal and three lateral, all spaced $2 \mathrm{~mm}$ apart ( 3 models were available with exploration lengths of: 34,42 and $50 \mathrm{~mm}$ ). These three tetrodes cover a theoretical triangular surface of $2 \mathrm{~mm}^{2}$. The three-tetrode configuration was generally chosen when the electrode passed through white matter between the lateral and distal cerebral targets, where it is usually not useful to record EEG signal.

These new hybrid electrodes have been under clinical investigation in our epilepsy center since 2015 , with an agreement to implant up to four hybrid electrodes per patient. 
A

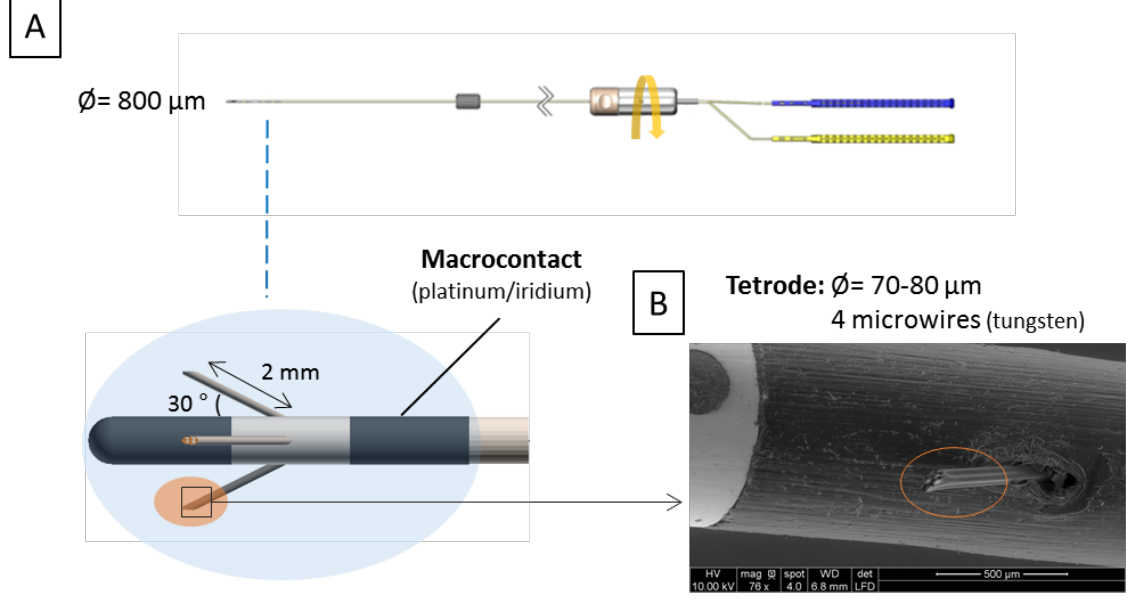

C

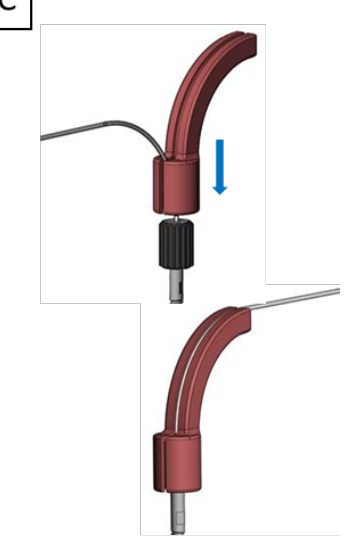

D
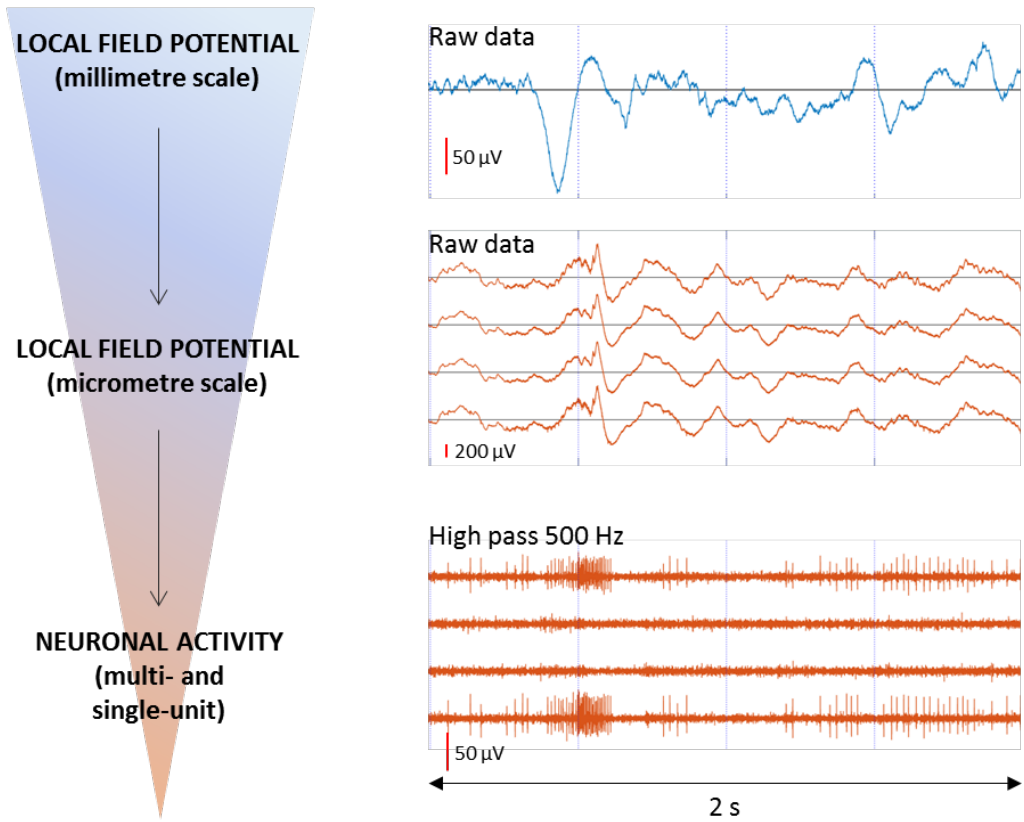

macro 1-2

High pass $500 \mathrm{~Hz}$

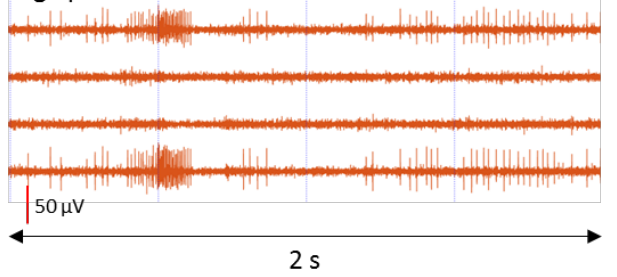

micro 1

micro 2

micro 3

micro 4

Figure 1: The new hybrid electrode. (A) The electrode contains two connectors, one for the macroelectrodes (in blue) and one for the microelectrodes (in yellow). The tetrodes can be extended with a micro-screw (yellow arrow) from the shaft between the most distal macrocontacts (macrocontacts 1-2) by up to $2 \mathrm{~mm}$. Depending on the model, two or three tetrodes can be extended. (B) Scanning Electron Microscopy of a tetrode partly extended from the shaft of a macroelectrode. (C) A sheath support is needed on the guide screw to ensure that the wires have an optimal curvature radius, and therefore, correct functioning of the input/output mechanism. (D) The design of these electrodes enables the multiscale 
recording of EEG, LFP, single and multi-unit activity. In blue: an interictal epileptiform discharge (i.e. an epileptic spike) recorded by a macrocontact (raw signal). Note the difference in morphology between the interictal epileptic spikes recorded simultaneously on the micro- and macroelectrodes. In orange: the same interictal epileptiform discharge recorded by the four microwires of a tetrode (raw signal) and the corresponding neuronal activity (high pass $500 \mathrm{~Hz}$ ).

\subsection{Neurosurgery}

Considering that the hybrid electrodes had the same diameter as the clinical electrodes, they did not require any change in the neurosurgical procedure. The rigidity of the clinical and hybrid electrodes in particular, remained the same. After the implantation of each hybrid electrode, a sheath support was placed on the guide screw to ensure that the cables had an optimal radius of curvature, resulting in correct functioning of the extension or retraction of the tetrodes (Fig. 1C). The sheath support slightly increased the safety distance to $5 \mathrm{~mm}$ instead of $3 \mathrm{~mm}$ between the entry point of each hybrid electrode and the other electrodes. In our center, the tetrodes were generally extended by $2 \mathrm{~mm}$ immediately after implantation in the neurosurgery room, or the patient's room, as long as the micrometer screw is not buried under the bandage.

\subsection{Electrode location}

The choice of electrode locations was based solely on pre-SEEG clinical observations and on hypotheses concerning the location of the seizure onset zone based on a non-invasive 
assessment, i.e. tailored implantation. The electrodes were implanted in brain regions chosen for clinical purposes only.

\subsection{Recordings}

Macrocontact signals were recorded using two SystemPLUS EVOLUTION 64-channel acquisition units (Micromed, France) at a sampling rate of 2,048 Hz (anti-aliasing filter: 926.7 $\mathrm{Hz}$; high-pass filter: $0.15 \mathrm{~Hz}$; low-pass filter: 1,000 Hz). Microelectrode signals were recorded using a 64-channel Cerebus System (Blackrock Microsystems, Salt Lake City, UT, USA) at a sampling rate of $30 \mathrm{kHz}$ (0.3-7.5 kHz bandwidth). Line noise cancellation at $50 \mathrm{~Hz}$ was applied. For patient 22, macroelectrode and microelectrode signals were both recorded using a 256channel Atlas System (Neuralynx, Bozeman, MT, USA). A macrocontact located in white matter was used as a reference for both systems.

Macrocontacts were recorded 24/day. Microelectrodes were recorded for 1 hour in five morning sessions while patients watched episodes of a TV show.

\subsection{Neuroimagery control}

The location of each electrode contact was based on a pre-operative 3D T1-weighted MRI/postoperative CT-scan data fusion. The resolution of this fusion allowed visual verification of the anatomical location of each contact and whether it was located in gray or white matter. This visual inspection was completed by normalization in the MNI anatomical space (Fig. 2). 
In order to investigate potential subclinical complications of electrode implantation such as tissue damage or bleeding along the electrode trajectory, a postoperative MRI was performed 3 to 6 months after the end of the SEEG and the explantation of the electrodes (Siemens/Philipps 3 Tesla, T2, FLAIR, and T2 gradient-echo based sequences (T2*)). A neurologist (JC) verified each postoperative MRI for subclinical injuries (i.e. not inducing any symptom) caused by electrode insertion (easily visible as a linear hypersignal on T2 and FLAIR sequences) and subclinical bleeding (hyposignal on T2 gradient echo-based sequences). We distinguished (1) clearly visible linear hyperintensities on T2 and FLAIR sequences that follow a part or the entire trajectory of the electrode; (2) hematomas, which correspond to a relatively well circumscribed pool of blood (with a mass effect at the acute phase, but small enough to remain subclinical); (3) "linear bleeding" which generally corresponds to a pseudolinear hyposignal on $\mathrm{T}^{*}$ that follows a part or the entire trajectory of the electrode. To our knowledge, this type of "linear bleeding" is not well described in the literature. It corresponds to the asymptomatic sequelae of electrode paths, which are well known and expected, but which, until now, have mainly been taken into account on T2 or Flair sequences. These sequences show only a linear cavity and gliosis but not possible hemorrhage. The magnetic inhomogeneity of iron and deoxyhemoglobin causes sustainably faster $\mathrm{T} 2$ * relaxation, leading to signal intensity loss on T2* MRI (Chavhan et al., 2009; Ko et al., 2018). Therefore, T2* MRI is highly sensitive in the detection of recent or old cerebral hemorrhages and T2 hyperintense foci most frequently highlight lesions involving white matter (Bekiesińska-Figatowska, 2004).

Please note that tetrodes could not be accurately localized using brain imagery, neither with a variety of MRI sequences and machines nor with X-ray imaging. This means that we do not know exactly where the tetrodes spread radially although we know the distance between 
the tetrodes and the adjacent macrocontacts as well as the precise distance between each tetrode (see above and Figure 1A for more information).

\subsection{Impedance}

Electrochemical Impedance spectroscopy (EIS) measurements were taken using a VMP3 Biologic potentiostat coupled with EClab Software. The impedance spectra were recorded by applying a $5 \mathrm{mV}$ root mean square (RMS) for the sine wave with frequencies varying from $1 \mathrm{~Hz}$ to $10 \mathrm{kHz}$. Measurements were taken by immersing tetrodes in artificial cerebrospinal fluid (ACSF) at $37^{\circ} \mathrm{C}$ for 24 days (Lecomte et al., 2017). Impedances at $1 \mathrm{kHz}$ were recorded every day. Due to recording failures, the impedance of microelectrodes 1,5 and 7 were not collected respectively on days 0,1 and 2 .

\subsection{Noise level}

We used a MATLAB toolbox (MicMac) developed in our lab, which allows simultaneous and synchronized macro and micro EEG signal visualization, to analyze EEG signals on different scales. We also measured the noise level and the changes in noise level throughout the days of recording. The noise level in an electronic system can be measured as the root mean square (RMS) voltage, which provides an overall measure of the magnitude of the recorded signal. We computed the RMS voltage on filtered signals $(300-3,000 \mathrm{~Hz})$ for each electrode for a 10 min period (bin duration: $60 \mathrm{~s})$. 


\subsection{Single-unit isolation}

Spike sorting was performed on one-hour daily recordings (maximum of five days per patients). We considered only the electrodes located in gray matter. Each microwire signal was bandpass filtered using a Butterworth filter $(300-3,000 \mathrm{~Hz})$ and whitened. We detected action potentials offline on each tetrode. Spike sorting was performed using SpyKING CIRCUS, which relies on a template matching-based algorithm (Yger et al.,2018). An action potential was detected if the amplitude was above a threshold of six times the median of the absolute deviation of the voltage. We took into account different quality metrics (interspike interval histograms and refractory period violations, scatterplots of the different clusters, action potential amplitude over time, autocorrelogram, crosscorrelogram and density plots) to optimize spike sorting (Hill et al., 2011). The resulting data were inspected manually and refined using the MATLAB graphical interface SpyKING CIRCUS.

\subsection{Electrical brain stimulation (EBS)}

EBS is a standard clinical procedure that is used to stimulate the brain areas sampled with the intracerebral electrodes in order to assess the local propensity of these brain areas to induce seizures, and to assess functional mapping before neurosurgery. In our center high frequency EBS at $50 \mathrm{~Hz}$ or low frequency EBS at $1 \mathrm{~Hz}$ (pulse duration: $1 \mathrm{msec}$, duration of EBS: from 5 seconds for high frequency EBS to 10-30 seconds for low frequency EBS) is applied in a bipolar manner to each contact in grey matter, with current intensity gradually increasing from 0.5 to $3 \mathrm{~mA}$ according to French SEEG guidelines (Isnard et al., 2018). The question of whether hybrid electrodes are compatible with this standard clinical procedure might be asked. We analyzed the stimulation procedures of all patients included in this study. 


\section{RESULTS}

A total of 240 clinical macroelectrodes (mean per patients \pm SD: $8.57 \pm 1.85$, range [512]) and 102 hybrid electrodes (mean per patient \pm SD: $3.6 \pm 0.62$, range [2-4]) were implanted in 28 patients (median age $=33.5$ years old; range [14-63]). Of the 102 hybrid electrodes, 69 had two tetrodes and 33, three tetrodes. The tetrodes were mainly placed in the medial temporal lobes but also in various other brain structures (Fig. 2).
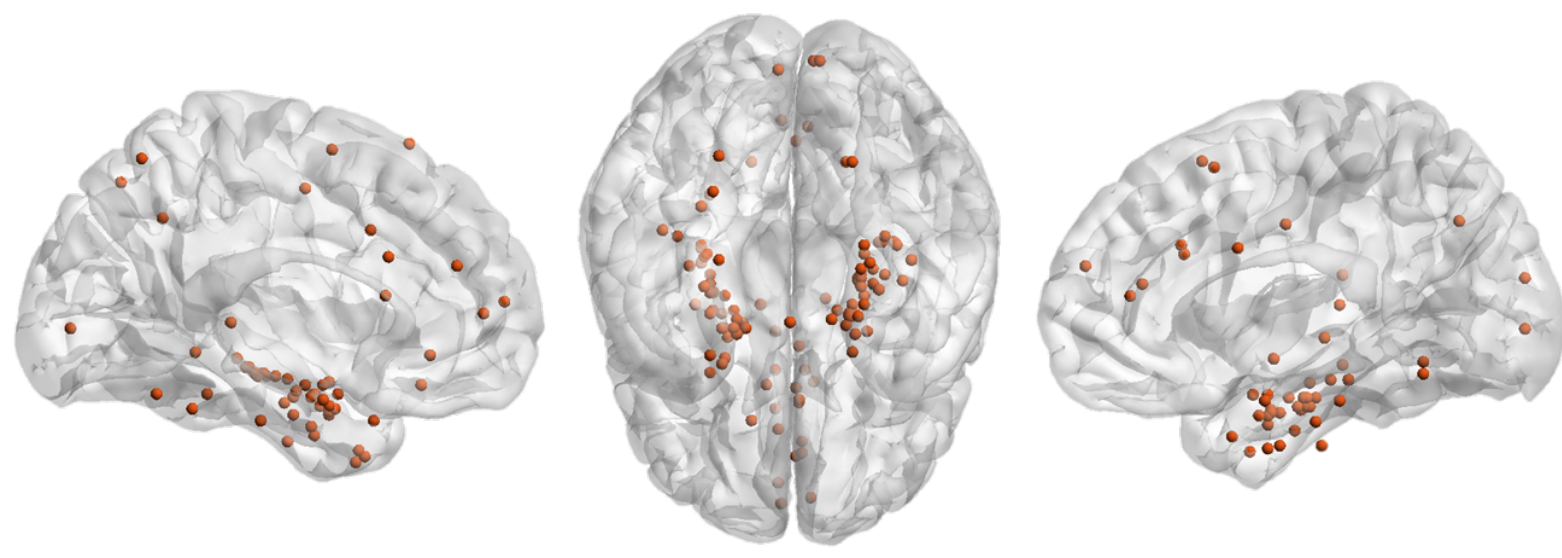

Figure 2 - Hybrid electrode locations in all patients. A total of 102 hybrid electrodes were implanted in 28 patients. The most distal contact of all the hybrid electrodes is depicted in a normalized glass brain after normalization in the MNI anatomical space (Xia et al.,2013). The tetrodes are adjacent to each of these contacts. 


\subsection{Safety of the new hybrid electrode}

The use of the hybrid electrode caused no adverse events or clinical complications such as hemorrhage, infection, or clinical deficit during or after this clinical trial in any of the 28 patients. A routine CT scan was performed several hours after implantation and after the recovery room to verify the absence of acute brain lesion (such as brain hemorrhage, subdural or extradural hematoma or brain edema). No specific lesion was linked to the hybrid electrodes on these CT scans.

In order to investigate subclinical complications caused by the electrodes (i.e., the percentage of hematomas, linear hyperintensities or linear bleeding on the ancient trajectories of the electrodes), fourteen patients (50\%) had a T2 and a T2*-weighted MRI scan at least 2 months after the removal of the hybrid electrodes (Fig. 3A). This corresponds to 116 clinical macroelectrodes and 47 hybrid electrodes. The remaining patients have not yet had a control MRI. The percentage of hematomas, linear hypersignals on the ancient trajectories of the electrodes, and linear bleeding (Hefft et al., 2013) was assessed by a neurologist (JC) who was blinded to the type of electrode (hybrid or clinical). Subclinical complication rates were similar in clinical and hybrid electrodes (Fig. 3B, Fisher's Exact Test for Count Data, T2/FLAIR linear hyperintensities, $P=1,95 \%$ confidence interval $=0.07-4.78$; hypersignal, $P=1,95 \%$ confidence interval $=0.25-2.47$; linear bleeding, $P=0.49,95 \%$ confidence interval $=0.61-$ 2.67). 


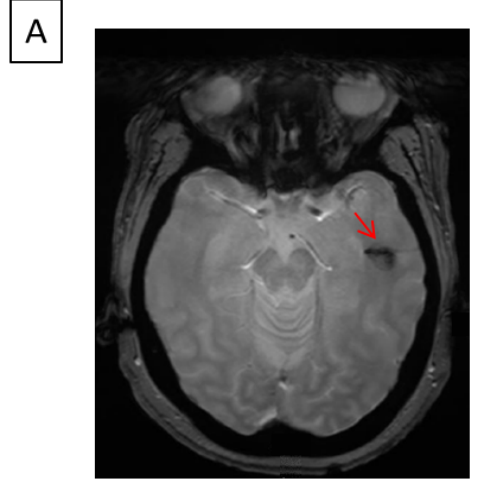

Hematoma

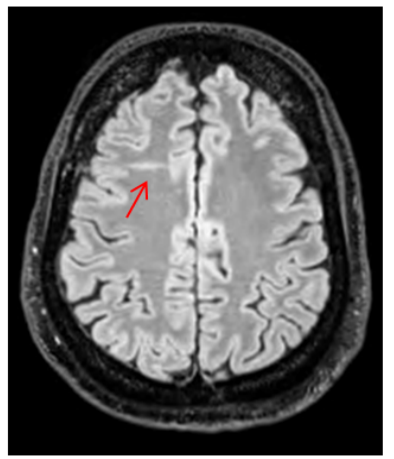

Linear

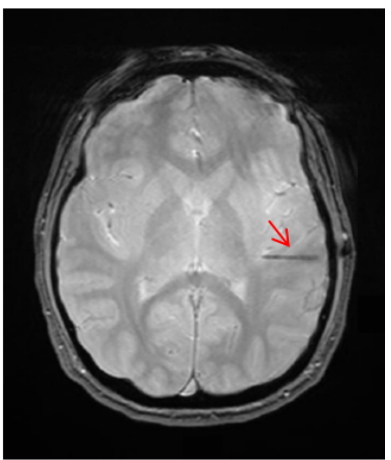

Linear

$B$

bleeding

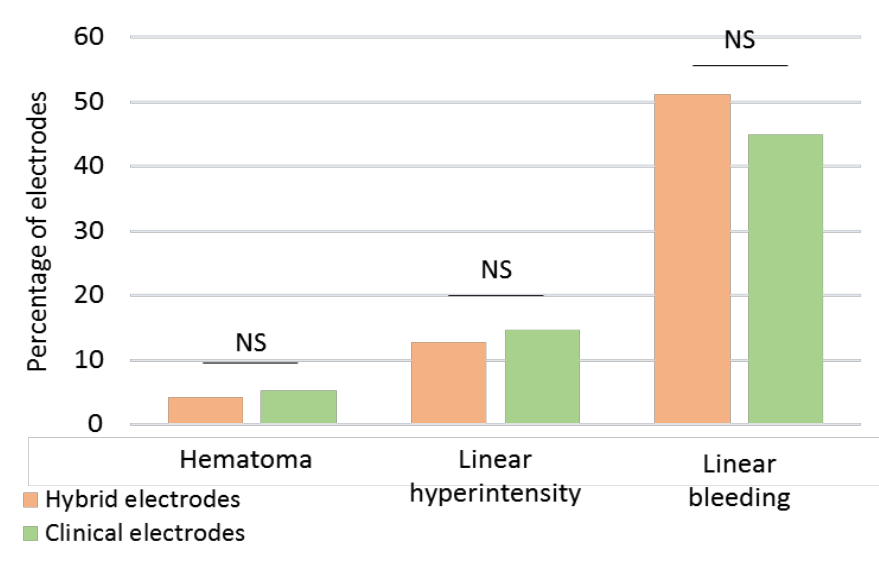

Figure 3 - The new hybrid electrode does not increase the number of subclinical

complications. (A) T2 and T2* MRI sequences showing typical examples of subclinical injuries caused by clinical and hybrid electrodes. (B) Percentage of hematomas, linear hyperintensities, and linear bleeding associated with the use of clinical macroelectrodes $(n=116)$ and hybrid electrodes $(n=47)$. No significant difference was observed between the two types of electrodes.

\subsection{Impedances}

Impedance was measured for four tetrodes (12 microelectrodes) immersed in ACSF for 24 days at $37^{\circ} \mathrm{C}$ (Fig. 4). The median impedance was $103 \mathrm{k} \Omega$ (range: $67-179$ ) for the first ten days after which it started to increase slightly until the twentieth day, with a median 
impedance of $224 \mathrm{k} \Omega$ (range: 129-285), (Friedman rank sum test, $\left.\mathrm{df}=14, P=8.91^{-16}\right)$. Of note, tetrodes are usually retracted around the $8^{\text {th }}$ day on our epilepsy unit.

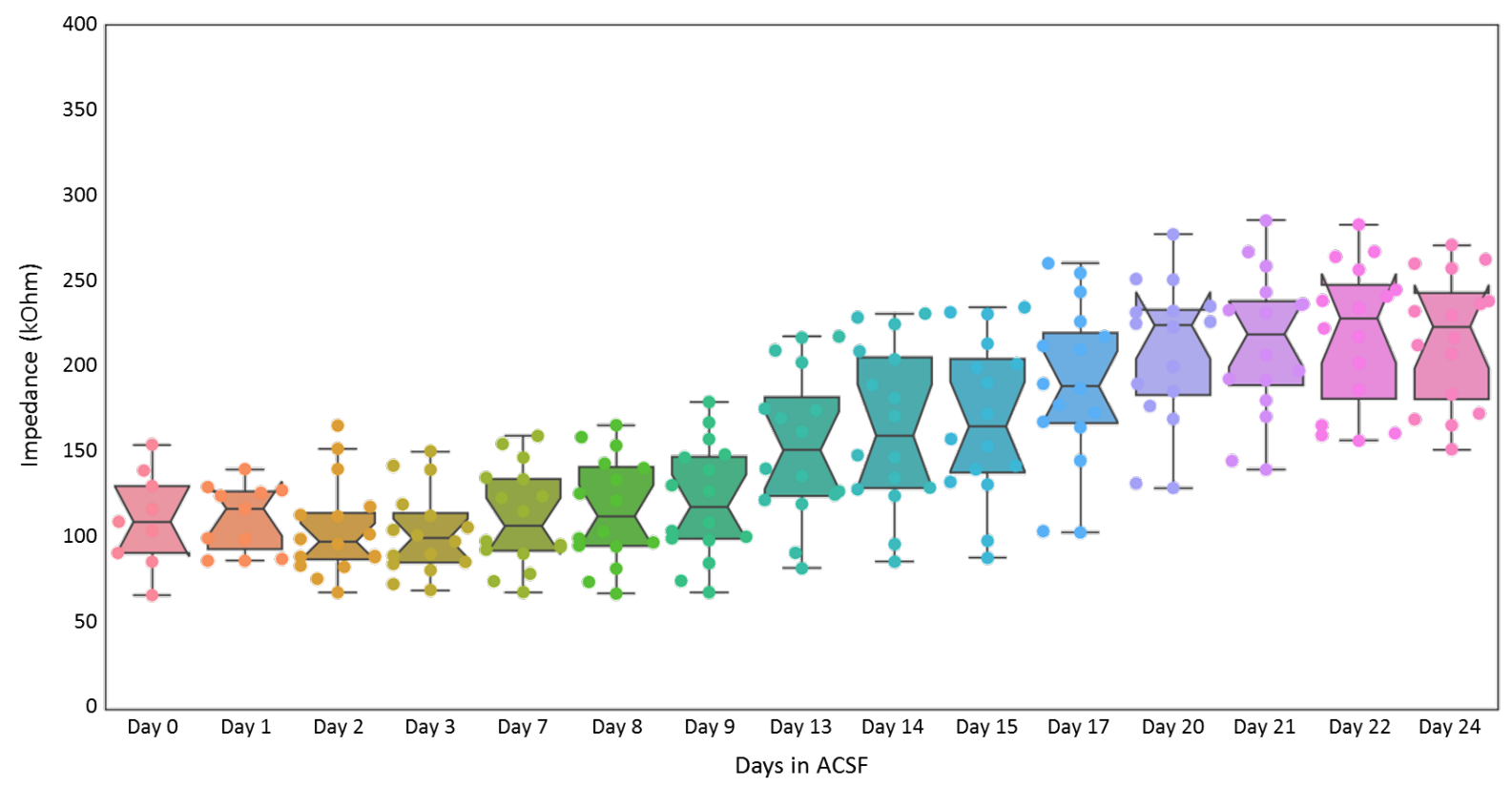

Figure 4 - Electrochemical impedances. Notched boxplot representing the changes in electrochemical impedances at $1 \mathrm{kHz}$ on four tetrodes immersed in ACSF for 24 days at $37^{\circ} \mathrm{C}$. Each point represents a single wire.

\subsection{Noise levels}

The noise level was measured on all hybrid electrodes from patients 10 to 28 during the 5 days of recording (the days were not necessarily consecutive because of the week-ends) (Fig. 5). No difference from the days after implantation was observed (median level $=22.25$ $\mu \mathrm{V}$; range: $14.87-52.19 \mu \mathrm{V}$; Friedman rank sum test, $\mathrm{df}=7, P=0.1386)$. 
Please note that the noise level may vary considerably from one setting to another as shown in Fig. 6, where comparisons were made between different amplifiers or with the same amplifier but different settings (a clinical room and a Faraday cage).

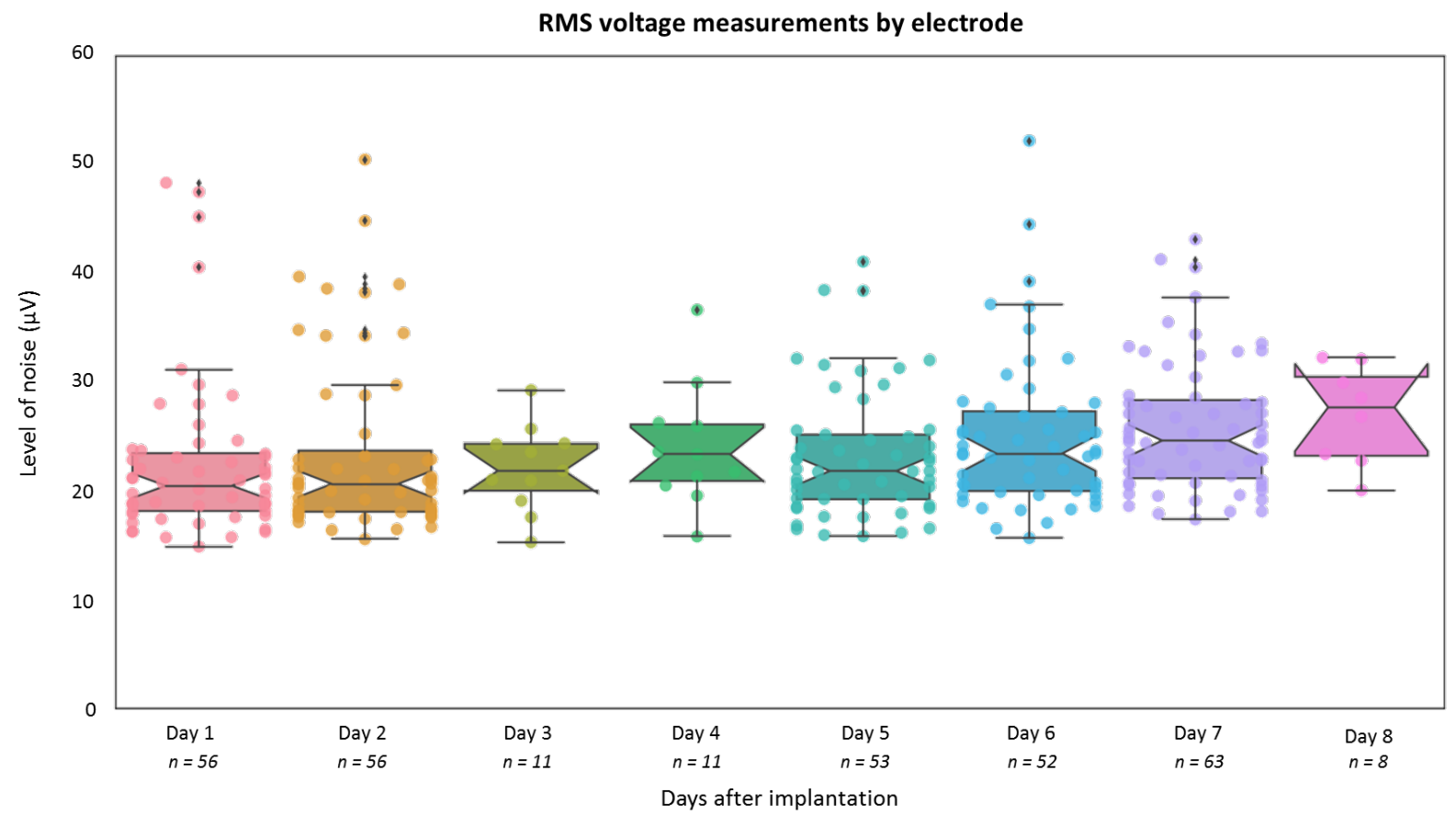

Figure 5 - Noise level. Change in noise amplitude (RMS voltage, filtered signal 300-3,000 Hz) on the recording days following implantation. Each point represents an electrode and the total number of electrodes is indicated below each day. It is important to note that the choice of recording system and the clinical conditions under which the recordings take place can have a significant impact on the noise level as shown in Fig. 6. 


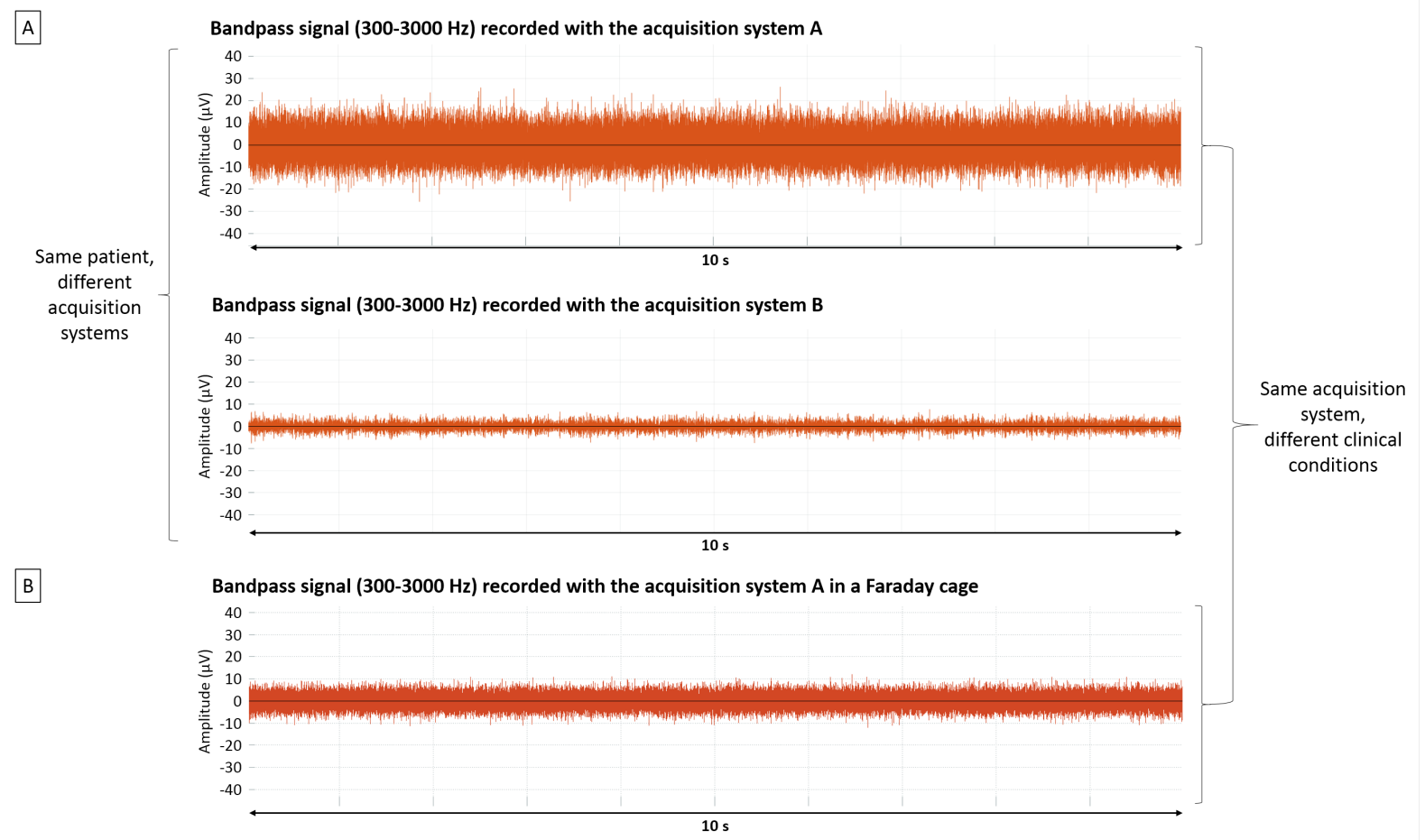

Figure 6-Comparison of different acquisition systems and clinical conditions. A) Filtered signal from a microwire located in the hippocampus of patient 22 and recorded a few minutes apart by two different acquisition systems, $A$ (Blackrock) and B (Neuralynx). Note that the referencing system is not the same in both cases, so they cannot be directly compared. B) Filtered signal recorded with the same acquisition system A (Blackrock) in two different patients and settings: in a room on our Epilepsy unit in Toulouse (top) or in a Faraday cage on the Epilepsy unit in Lyon (bottom).

\subsection{Multiscale events}

The fact that the tetrodes extended between two macrocontacts enabled simultaneous multiscale recordings of the same brain region (Fig. 1C). Signals can be recorded on different scales (such as between the adjacent macrocontacts and tetrodes, but also between the tetrodes themselves), and may reveal different information. An example is that 
interictal epileptiform discharges may only be detected by microelectrodes (Fig. 7A) or have a different temporal and morphological structure between micro- and macroelectrodes (Fig. 1D). Microelectrodes also enabled the detection of additional focal events such as fast ripples which are considered to be new biomarkers of the seizure onset zone (Zijlmans et al., 2017; Roehri et al., 2018) (Fig. 7B). It is interesting to note that even if the three tetrodes of an electrode are localized in a confined space, they may not detect the same events, or not at the same latency. For example, a fast ripple may only be seen on one or two tetrodes or sometimes can be delayed on one tetrode in relation to the others (Fig. 8).
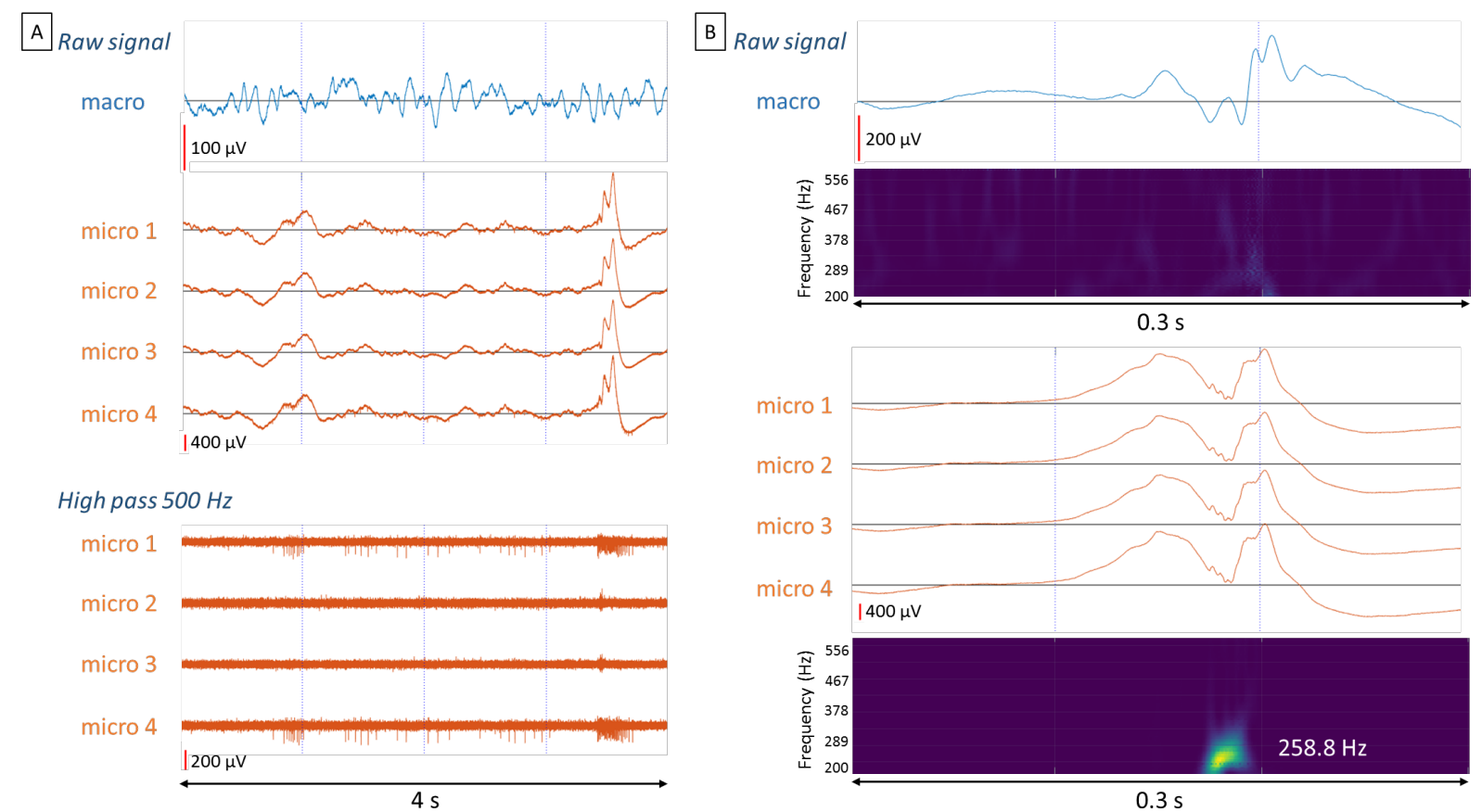

Figure 7 - Contributions of microelectrode recordings. (A) An interictal epileptic discharge (IED) associated with increased neuronal activity was observed with microelectrodes while no IED could be detected with the nearby macroelectrodes. The electrode was located in the hippocampus of patient 6. (B) Example of an IED recorded both on the macro and 
microelectrodes. However, fast ripples $(200-600 \mathrm{~Hz})$ were visible only in the microelectrode recordings. The electrode was located in the hippocampus of patient 6.

A

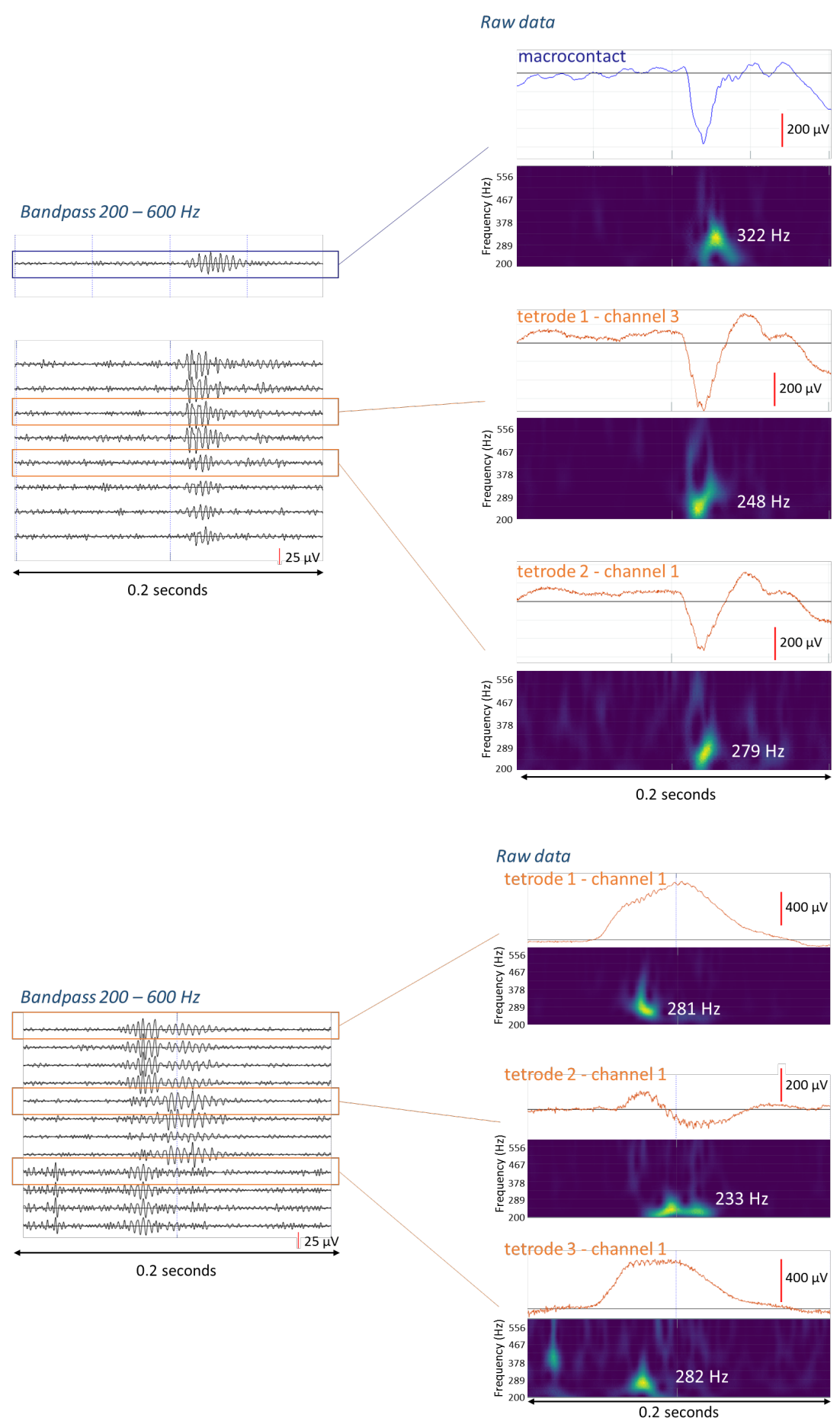

Figure 8-A) Different features and time of onset between macroelectrodes and tetrodes of the same hybrid electrode. Examples of raw and filtered signals (bandpass: $200-600 \mathrm{~Hz}$ ) 
recorded on each tetrode of an electrode located in the amygdala and on adjacent macrocontacts (patient 18). The fast-ripples do not appear exactly at the same time on the macro and on the microelectrodes and their frequencies differ. B) Fast ripples are localized in a confined space. Examples of raw and filtered signals (bandpass: $200-600 \mathrm{~Hz}$ ) recorded on each tetrode of an electrode located in the hippocampus (patient 20). The fast ripples are out-of-phase on the different tetrodes, especially on the second tetrode in comparison to the others.

\subsection{Electrical brain stimulation (EBS)}

The implantation of the hybrid electrodes did not impact EBS, neither on the two macrocontacts surrounding the tetrodes nor on remote contacts. It was possible to use the tetrodes to record LFP and single-unit activity dynamics induced by EBS, before and after EBS artifacts (corresponding to the train of pulses) (Fig. 9).
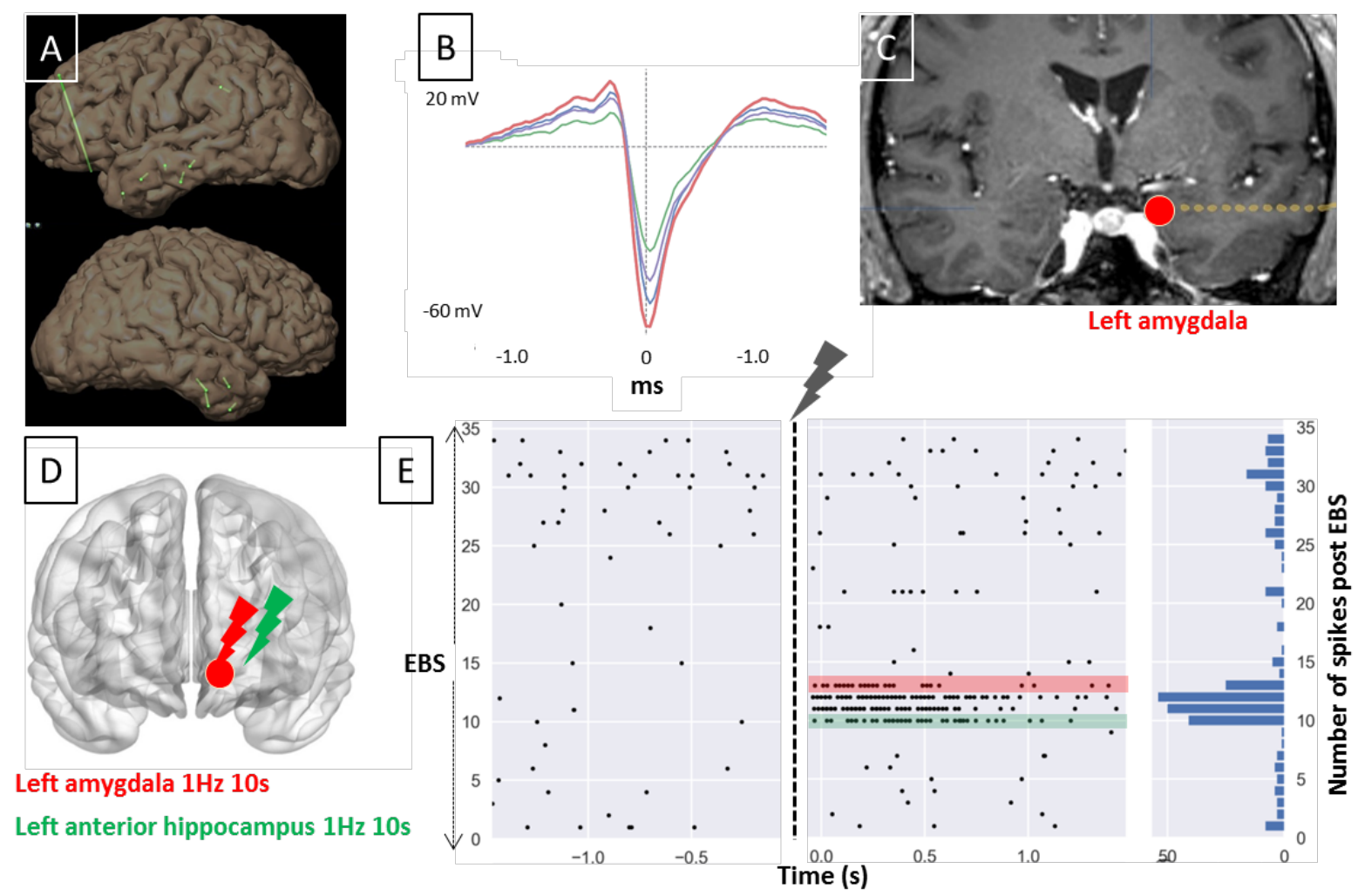
Figure 9-Example of neuronal activity recorded after EBS. (A) Diagram of the electrodes implanted in the brain of patient 18. (B) A neuron was recorded in the amygdala. Each curve corresponds to the waveform template of this neuron on a different microwire of the tetrode. (C) Illustration of the electrode trajectory toward the amygdala. The tetrodes are located in the red circle. (D) Schematic representation of two stimulations (at $1 \mathrm{~Hz}$ for $10 \mathrm{~s}$ ), one in the amygdala (red) were the neuron presented in B was located and one in the hippocampus (green), far from the neuron. (E) Representation of the spiking activity of the neuron in $B$ before and after 34 different EBS. The EBS in the amygdala (red-local) and in the hippocampus (green - distant) increased the activity of this neuron. The activity during stimulation is not shown due to saturation of the amplifier related to stimulation artifacts.

\subsection{Neuronal yield}

Patients 1-11 were excluded from the analysis due to interference between the microwire and macrowire acquisition systems that created an artifact at $2,048 \mathrm{~Hz}$ (the microelectrodes were recorded on the Blackrock system while the macroelectrodes were recorded in parallel on our Micromed clinical system, (see Methods). This problem was solved when the Micromed amplifiers were run on batteries). Patients 17 and 22 were also excluded because the SEEG ended prematurely and because the signal was acquired with another acquisition system (patient 22).

We analyzed the signal recorded by 30 electrodes (68 tetrodes) in five days. On average, we were able to record $2.08 \pm 2.86$ single neurons per tetrode per day on the tetrodes that recorded neuronal activity. In most cases, the tetrodes that did not record activity were located in white matter. Single neurons were recorded on $60 \%$ of the tetrodes. Neuronal 
activity could be recorded on $71 \%$ of the tetrodes when multi-unit activity was taken into account. We were able to detect a maximum of 13 single neurons with a single tetrode located in the amygdala of patient 14 . The number of neurons per tetrode was $>6$ neurons in $32 \%$ of the tetrodes, and it is of note that most were in the amygdala (Fig. 10). We also assessed whether the neuronal yield depended on the regions under investigation for the regions that were the most often recorded. On average, it was in the amygdala that we recorded the most neurons $(3.68, S D=3.20)$, followed by the entorhinal region $(3.45, S D=3.98)$. In contrast, we recorded comparatively fewer neurons in the hippocampus (head of the hippocampus: 0.95, $S D=1.61$, tail of the hippocampus: $1.75, S D=2.69)$. In our study, the global neuronal yield remained fairly constant over the days of recording (up to 8 days post-implantation). However, we have no way of knowing whether it was the same units that were recorded as recordings took place during different sessions rather than continuously. 


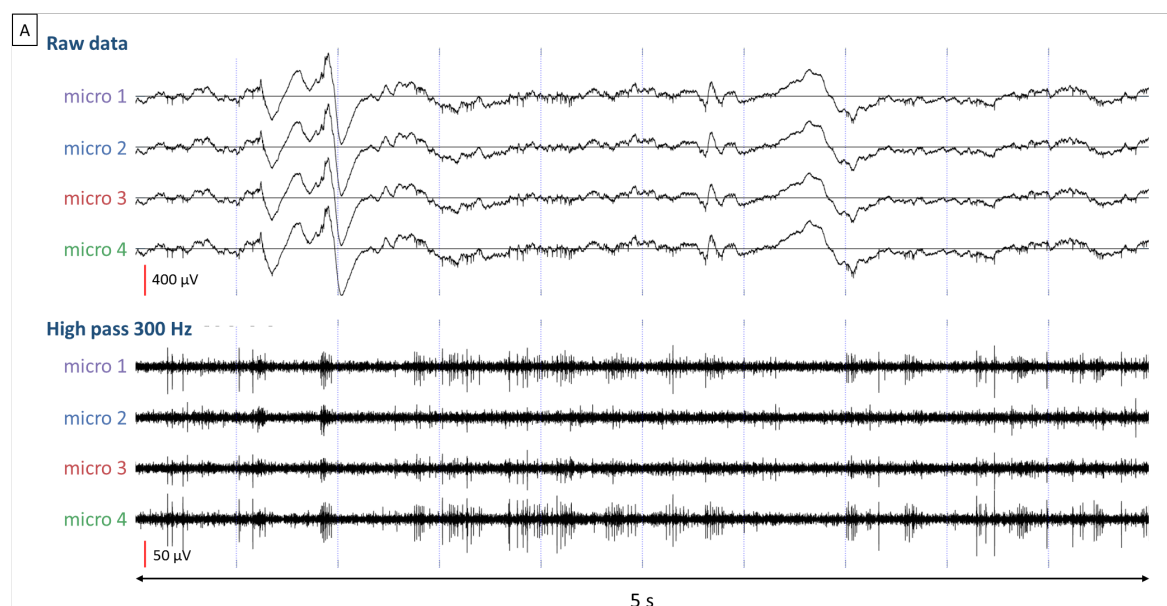

B

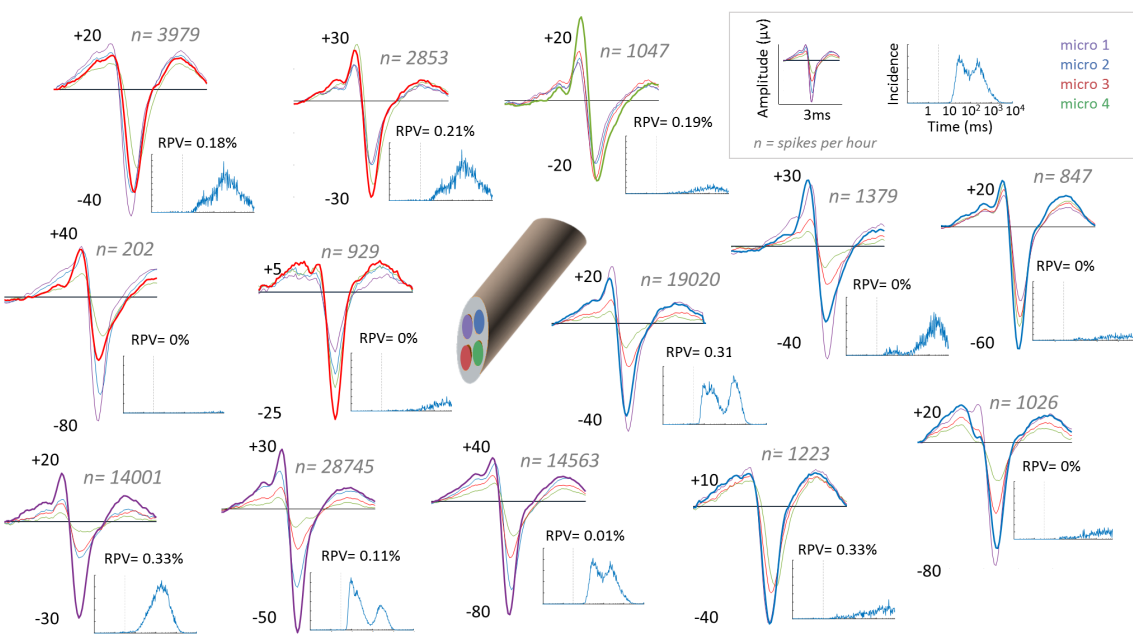

c

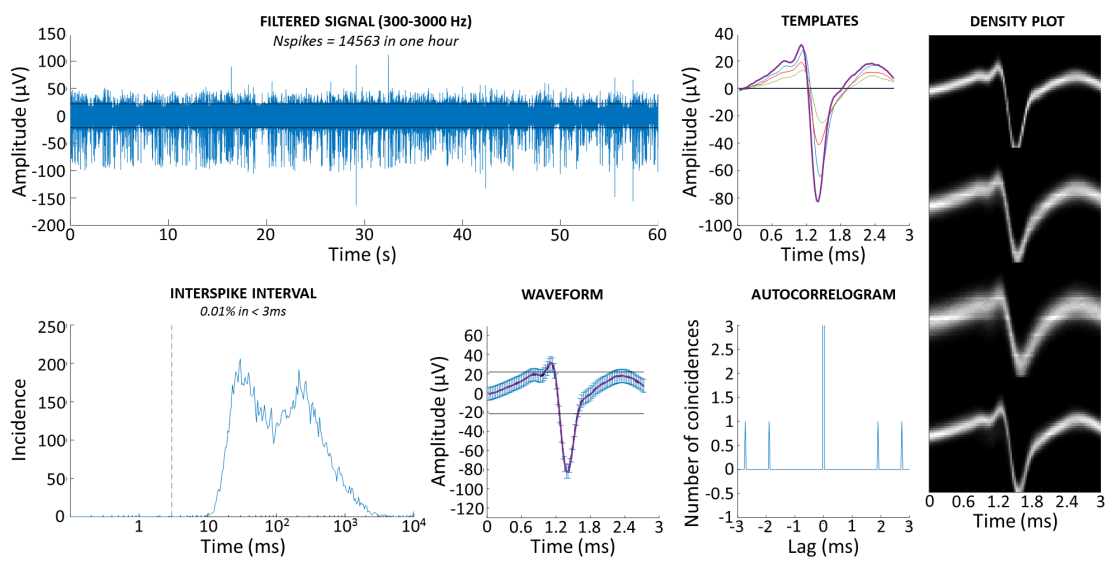

Figure 10 - Neuronal spiking activity. (A) Raw and filtered signal (high pass: $300 \mathrm{~Hz}$ ) recorded from a tetrode located in the amygdala of patient 14. (B) Thirteen neurons were isolated from a one-hour recording in this patient. The curves correspond to the average waveform detected on each wire of the tetrode. Each waveform plot also shows the magnitude of the waveform in microvolts and the number of spikes recorded by the tetrode. The interspike 
interval was computed for each neuron. (C) These are different metrics to assess neuronal quality for one unit: spike visibility on filtered signal (300-3000 Hz); template appearance on the four microwires of the same tetrode; density plot on the four microwires, the interspike interval histogram; mean \pm SEM (standard error of mean) of the waveforms (computed from the microwire with the widest signal); autocorrelogram.

\section{DISCUSSION}

In this study, we present a new hybrid electrode equipped with tetrodes which enables simultaneous analysis of intracerebral activity on macro- and micro-scales in epileptic patients.

In comparison to standard clinical macroelectrodes, the use of new hybrids electrodes caused no particular or additional clinical complications or adverse events. They also did not cause more subclinical brain damage (i.e. hematomas, linear bleeding or more tissue damage at electrode insertion sites) than clinical macroelectrodes. The same conclusions were reached with another type of hybrid electrodes with a bundle of wires, which appeared to be as safe as standard depth electrodes (Fried et al. 1999; Hefft et al. 2013; Carlson et al. 2018). Therefore, in general, hybrid electrodes, including the one presented in this study, appear to be as safe as clinical electrodes.

Please note that we report on "linear bleeding" in $50 \%$ of our patients. To our knowledge, this type of linear bleeding is not well described in the literature. It corresponds to the sequelae of electrode paths which are well known, inevitable and expected, but which, until now, have mainly been taken into account on $\mathrm{T} 2$ or Flair sequences. These sequences only show a linear cavity and gliosis but not the possibility of hemorrhage. The main reason for this 
is probably a lack of focus by clinicians and neuroimagers on this type of anomaly, because they are perfectly asymptomatic and do not evolve with time. Furthermore, $\mathrm{T} 2 *$ sequences are not systematically performed if no research protocol and surgery are scheduled. Finally, 3T MRI scans have been performed in most of our patients with a much better resolution than has been reported in studies of only a few years ago. Therefore, overall, we highlight asymptomatic sequelae which have probably been largely overlooked until now.

Several types of device now make it possible to record brain electrophysiological activity in humans, including needle hybrid electrodes such as the one used in this study, grids and multi-electrode arrays. An adequate comparison of the safety of the different types of electrodes and models remains to be done. One pending question in particular is whether the smaller diameter of the Dixi electrode $(0.8 \mathrm{~mm})$ is of any consequence compared to the larger diameter of other needle electrodes, which can reach up to $1.3 \mathrm{~mm}$.

In our clinical study, these hybrid electrodes also had a minimal impact on the daily routine of SEEG exploration. However, we found that special care had to be taken not to bend the cable running from the electrodes to the connectors with the bandage. Special attention must also be paid to the electromagnetic environment of the room and the way in which the different cables are arranged in order to avoid electronic interference. In this article, we also show that the general noise level can vary significantly depending on the amplifiers and setup. This means that special care and effort should be devoted to attaining a suitable noise level. The most significant change for us was to decide which electrode insertion path plan should be used for the hybrid electrodes. This was because the spacing between the macrocontacts of hybrid electrodes is different from that of clinical electrodes and the number of macrocontacts is limited according to the model (see Methods section). In addition, these new 
hybrid electrodes are not MRI-compatible as they are made of tungsten. However, that did not affect our clinical routine because we use a CT scan after implantation to verify the absence of bleeding. Overall, these hybrid electrodes were easily adopted by our team of epileptologists and our neurosurgeon, with no increase in the duration of the implantation.

Impedance can greatly affect the quality of neuronal recordings as the ability to record action potentials from individual neurons is dependent on a tradeoff between the geometric area of the tip of the recording instrument and the impedance of the tip (Ludwig et al., 2006). A small geometric area enables isolation of action potentials from distant neural sources (Drake et al., 1988; Humphrey and Schmidt, 1990; Paik et al.,2003). Usually, a decrease in geometric area causes an increase in impedance (Humphrey and Schmidt, 1990; Paik et al., 2003). Lowering the impedance could improve neuronal recording quality by increasing the signal-to-noise ratio (Ferguson et al., 2009). The expected impedance for the microwires of a tetrode between 10 and $30 \mu \mathrm{m}$ in diameter is $100-500 \mathrm{kOhm}$ (at $1 \mathrm{kHz}$ ) (Ferguson et al., 2009). Similarly, the impedance of multi-wire electrodes with a diameter of 12 to $25 \mu \mathrm{m}$ is usually approximately $100 \mathrm{kOhm}$ (Hill et al., 2011). In our study, the diameter of each microwire was $20 \mu \mathrm{m}$ and the impedance was approximately $100 \mathrm{kOhm}$ during the first ten days. The impedance slightly increased to $220 \mathrm{kOhm}$ after 3 weeks in vitro. Overall, the impedances we measured remained within reported standards.

It is crucial to obtain a minimal noise level in order to detect a larger number of units in the recordings, as only units with amplitudes above the noise level can be detected. Our median noise level was $22.25 \mu \mathrm{V}$. This noise level appears to be satisfactory as we were able to record single units. However, our results were acquired in suboptimal recording conditions and the quality of the signal could be improved. In fact, the noise level observed in other 
centers is sometimes lower. For example, Rutishauser et al. (2015) were able to detect units with an amplitude of $15 \mu \mathrm{V}$, whereas we had difficulty detecting units with amplitudes below 20-25 $\mu \mathrm{V}$. There are several possible explanations for the difference between centers: the quality of the electromagnetic environment, the quality of the cables between the tetrodes and the headstage and the quality of the amplifier, all of which should be optimized in the future.

In a model of hybrid electrodes that is already commercialized, single neurons were observed on $40 \%$ of the microwires, and a bundle of 8 microwires recorded an average of 2.1 \pm 0.9 single neurons (Misra et al., 2014). With more experience, $70-80 \%$ of the bundles of microwires could record neurons (Carlson et al., 2018). Carlson et al. also reported that the number of neurons per bundle varies widely, from 0 to 16 , possibly according to the brain region explored. For example, the amygdala appears to be a brain region where it is relatively easy to record a large number of neurons.

The tetrode configuration was introduced in animal research in the early nineties and has been widely used since (Gray et al., 1995; Lévesque et al., 2016; 2018; O’Keefe and Recce, 1993; Wilson and McNaughton, 1993). It facilitates the isolation of single units. To our knowledge, the maximum number of single neurons that have been isolated with one tetrode in animals was 9 (Gray et al., 1995), although tetrodes have the potential to record many more neurons simultaneously (Buzsáki, 2004).

The number of neurons recorded on each tetrode in the current study varied widely, from 0 to $>10$. We observed single neurons on $60 \%$ of the tetrodes and we were able to record 2.08 \pm 2.86 single neurons per tetrode, per day. Taking into account multiple unit activity, up to $70 \%$ of the tetrodes showed some level of activity. This level of activity persisted with no 
obvious difference over time (in days) after implantation (from day $+1-$ day +8 ). With the new hybrid electrode, we were able to isolate up to 13 neurons with one tetrode, which seems promising and demonstrates the potential of this new hybrid electrode. Given our suboptimal recording conditions mentioned above, we think it will be possible to improve the neuronal yield once the signal-to-noise ratio of the recordings is improved.

With regard to this, there may still be more work to do to validate and compare different spike-sorting software as it is unclear whether they can be optimized in comparison to older software and whether they use the spatial information provided by the tetrode configuration optimally (Chaure et al., 2018). Spike sorting remains a field in which some level of subjectivity remains despite useful guidelines (Hill et al., 2011). New spike sorting software is being developed (e.g. the one we are using - SpyKing Circus - by Yger et al., 2018) and progress can be expected in the years to come.

Another important specificity of hybrid electrodes, which is unique to our 2 or 3 tetrodes per electrode configuration, is that the tetrodes can be extended from the shaft between the most distal macrocontacts by up to $2 \mathrm{~mm}$, and the distance between the macrocontacts and the tetrode tips is known with relative precision. This is particularly suitable for multiscale analysis, as both macro and microelectrodes are located in the same target brain region. Therefore, it is possible to record different signals within the same area such as: (1) the local field potential between the two macrocontacts adjacent to the tetrodes; (2) the local field potential of each tetrode in relation to either a distant contact (suitable for the recording of "larger" events) or to another tetrode (for "smaller", more local, events). Theoretically, the surface covered by or the distance between tetrodes can be determined and can therefore be used to approximate the size of the patch of cortex from which the electrophysiological events 
of interest originate. Of course, multiple and single-unit activities are recorded the same time. Using such schemes, it is possible to uncover neurophysiopathological events that are recorded on the tetrodes but not on the macrocontacts, such as local interictal epileptic discharge (Figure 7A) and fast ripples (Figure 7B). Events can also be recorded on a smaller scale, such as fast ripples occurring on two tetrodes but not on the third (Despouy et al., 2019), or different dynamics on each tetrode (Figure 8).

In conclusion, the design of this new hybrid electrode is different from that of other available hybrid electrodes implanted in humans. These electrodes are especially promising for conducting clinical multiscale analyses (of interictal epileptic discharges, high frequency oscillations and seizure onset or propagation), without changing the implantation procedure (Schevon et al., 2019). They offer the possibility to provide unique information regarding neurophysiopathological aspects of epilepsy or the physiological substrates of cognition. We showed that these electrodes are safe and work satisfactorily. At present, two or three tetrodes can be extended by up to $2 \mathrm{~mm}$. Further improvements are planned, such as extending tetrodes further to $3 \mathrm{~mm}$. The possibility of moving tetrodes over such a range with the micrometer screw will improve the neuronal search, which in turn may considerably improve the neuronal yield. Another possibility is to have tetrodes protruding from different levels of the macroelectrode, which would allow recordings from lateral parts of the cortex and the possibility to study brain regions that, so far, have been poorly explored in humans.

\section{ACKNOWLEDGEMENTS}

We would like to thank Pierre Yger for helping us to understand the technical features of SpyKING CIRCUS, Karine Bouyer for managing the electrodes, and Sophie Muratot and Simona 
Celebrini for their help in acquiring the data. We are thankful to the team from the Hospices Civils de Lyon (Lyon University Hospital Center) for their help with Figure 6 (Prof. S. Rheims funding from the IHU CESAME in the "Investissements d'Avenir" program - ANR-10- IBHU0003). We would also like to thank all the people involved, at one point or another, in the EPIFAR project.

\section{COMPETING INTERESTS STATEMENT}

ED is funded through a CIFRE industrial research agreement (no. 2015/1135) signed between DIXI Medical and the Centre de Recherche Cerveau et Cognition (CerCo). The remaining authors have no conflicts of interest.

FUNDING

This work received funding from the European Research Council, under the European Union Seventh Framework Programme (FP/2007-2013) / ERC Grant Agreement no. 323711 (the M4 project), the Fondation Française pour la Recherche sur l'Epilepsie (Prix Marion Clignet), the Fédération Française sur le Cerveau / Rotary Club, and a call for research projects in technological and organizational innovation (Toulouse University Hospital).

\section{REFERENCES}


Alvarado-Rojas C, Lehongre K, Bagdasaryan J, et al. Single-unit activities during epileptic discharges in the human hippocampal formation. Front Comput Neurosci 2013;7:140. https://doi.org/10.3389/fncom.2013.00140.

Babb TL, Carr E, Crandall PH. Analysis of extracellular firing patterns of deep temporal lobe structures in man. Electroencephalogr Clin Neurophysiol 1973;34:247-57.

https://doi.org/10.1016/0013-4694(73)90252-6.

Behrens E, Zentner J, van Roost D, Hufnagel A, Elger CE, Schramm J. Subdural and depth electrodes in the presurgical evaluation of epilepsy. Acta Neurochir 1994;128:84-7. https://doi.org/10.1007/bf01400656.

Bekiesińska-Figatowska M. T2-hyperintense foci on brain MR imaging. Med Sci Monit 2004;10:80-97.

Bower MR, Buckmaster PS. Changes in granule cell firing rates precede locally recorded spontaneous seizures by minutes in an animal model of temporal lobe epilepsy. J Neurophysiol 2008;99:2431-2442. https://doi.org/10.1152/jn.01369.2007.

Buzsáki G. Large-scale recording of neuronal ensembles. Nat Neurosci 2004;7:446-451. https://doi.org/10.1038/nn1233.

Capraz IY, Kurt G, Akdemir Ö, Hirfanoglu T, Oner Y, Sengezer T, et al. Surgical outcome in patients with MRI-negative, PET-positive temporal lobe epilepsy. Seizure 2015;29:63-8. https://doi.org/10.1016/j.seizure.2015.03.015.

Carlson AA, Rutishauser U, Mamelak AN. Safety and utility of hybrid depth electrodes for seizure localization and single-unit neuronal recording. Stereotact Funct Neurosurg. 2018;96(5):311-319. https://doi.org/10.1159/000493548.

Chaure FJ, Rey HG, Quian Quiroga R. A novel and fully automatic spike-sorting implementation with variable number of features. J Neurophysiol 2018;120:1859-71. https://doi.org/10.1152/jn.00339.2018. 
Chavhan GB, Babyn PS, Thomas B, Shroff MM, Haacke EM. Principles, Techniques, and Applications of T2*-based MR Imaging and Its Special Applications. RadioGraphics 2009;29:1433-49. https://doi.org/10.1148/rg.295095034.

Dalic L, Cook M. Managing drug-resistant epilepsy: challenges and solutions. Neuropsychiatr Dis Treat 2012;12:2605-16. https://doi.org/10.2147/NDT.S84852.

Despouy E, Curot J, Denuelle M, Deudon M, Sol J-C, Lotterie J-A, et al. Neuronal spiking activity highlights a gradient of epileptogenicity in human tuberous sclerosis lesions. Clin Neurophysiol 2019;130:537-47. https://doi.org/10.1016/j.clinph.2018.12.013.

Drake KL, Wise KD, Farraye J, Anderson DJ, BeMent SL. Performance of planar multisite microprobes in recording extracellular single-unit intracortical activity. IEEE Trans Biomed Eng 1988;35:719-32. https://doi.org/10.1109/10.7273.

Eissa TL, Dijkstra K, Brune C, Emerson RG, van Putten MJAM, Goodman RR, et al. Cross-scale effects of neural interactions during human neocortical seizure activity. Proc Natl Acad Sci 2017;114:10761-6. https://doi.org/10.1073/pnas.1702490114.

Ferguson JE, Boldt C, Redish AD. Creating low-impedance tetrodes by electroplating with additives. Sens Actuators Phys 2009;156:388-93. https://doi.org/10.1016/j.sna.2009.10.001.

Fried I, MacDonald KA, Wilson CL. Single Neuron Activity in Human Hippocampus and Amygdala during Recognition of Faces and Objects. Neuron. 1997;18:753-65. https://doi.org/10.1016/S0896-6273(00)80315-3.

Fried I, Wilson CL, Maidment NT, Engel J, Behnke E, Fields TA, et al. Cerebral microdialysis combined with single-neuron and electroencephalographic recording in neurosurgical patients: Technical note. J Neurosurg 1999;91:697-705.

https://doi.org/10.3171/jns.1999.91.4.0697.

Gray CM, Maldonado PE, Wilson M, McNaughton B. Tetrodes markedly improve the reliability and yield of multiple single-unit isolation from multi-unit recordings in cat striate cortex. J Neurosci Methods 1995;63:43-54. https://doi.org/10.1016/0165-0270(95)00085-2. 
Hefft S, Brandt A, Zwick S, von Elverfeldt D, Mader I, Cordeiro J, et al. Safety of Hybrid Electrodes for Single-Neuron Recordings in Humans: Neurosurgery 2013;73:78-85. https://doi.org/10.1227/01.neu.0000429840.76460.8c.

Henze DA, Borhegyi Z, Csicsvari J, Mamiya A, Harris KD, Buzsáki G. Intracellular Features Predicted by Extracellular Recordings in the Hippocampus In Vivo. J Neurophysiol 2000;84:390-400. https://doi.org/10.1152/jn.2000.84.1.390.

Hill DN, Mehta SB, Kleinfeld D. Quality Metrics to Accompany Spike Sorting of Extracellular Signals. J Neurosci 2011;31:8699-705. https://doi.org/10.1523/JNEUROSCI.0971-11.2011.

Humphrey DR, Schmidt EM. Extracellular Single-Unit Recording Methods. In: Neurophysiological Techniques, II. New Jersey: Humana Press; 1990. p. 1-64. Available from: http://link.springer.com/10.1385/0-89603-185-3:1

Isnard J, Taussig D, Bartolomei F, Bourdillon P, Catenoix H, Chassoux F, et al. French guidelines on stereoelectroencephalography (SEEG). Neurophysiol Clin 2018;48:5-13. https://doi.org/10.1016/j.neucli.2017.11.005.

Kandel ER, Spencer WA. Electrophysiology of hippocampal neurons: II. After-potentials and repetitive firing. J Neurophysiol 1961;24:243-59. https://doi.org/10.1152/jn.1961.24.3.243.

Keller CJ, Truccolo W, Gale JT, Eskandar E, Thesen T, Carlson C, et al. Heterogeneous neuronal firing patterns during interictal epileptiform discharges in the human cortex. Brain 2010;133:1668-81. https://doi.org/10.1093/brain/awq112.

Ko HC, Ryu C-W, Yun SJ, Koh JS, Shin HS, Kim EJ. Characteristic Signs on T2*-Based Imaging and Their Relationship with Results of Reperfusion Therapy for Acute Ischemic Stroke: A Systematic Review and Evidence to Date. Neurointervention 2018;13:90-9.

https://doi.org/10.5469/neuroint.2018.01039.

Kreiman G, Fried I, Koch C. Single-neuron correlates of subjective vision in the human medial temporal lobe. Proc Natl Acad Sci 2002;99:8378-83.

https://doi.org/10.1073/pnas.072194099. 
Kwan P, Schachter SC, Brodie MJ. Drug-Resistant Epilepsy. N Engl J Med 2011;8. https://doi.org/10.1056/NEJMra1004418.

Lambrecq V, Lehongre K, Adam C, Frazzini V, Mathon B, Clemenceau S, et al. Single-unit activities during the transition to seizures in deep mesial structures: Seizures and Single-Unit Activities. Ann Neurol 2017;82:1022-8. https://doi.org/10.1002/ana.25111

Laxer KD, Trinka E, Hirsch L, Cendes F, Langfitt J, Delanty N, et al. The consequences of refractory epilepsy and its treatment. Epilepsy Behav 2014;37:59-70. https://doi.org/10.1016/j.yebeh.2014.05.031

Lecomte A, Degache A, Descamps E, Dahan L, Bergaud C. In vitro and in vivo biostability assessment of chronically-implanted Parylene C neural sensors. Sens Actuators B Chem 2017;251:1001-8. https://doi.org/10.1016/j.snb.2017.05.057.

Lévesque M, Herrington R, Hamidi S, Avoli M. Interneurons spark seizure-like activity in the entorhinal cortex. Neurobiol Dis 2016;87:91-101.

https://doi.org/doi:10.1016/j.nbd.2015.12.011.

Lévesque M, Chen LY, Hamidi S, Avoli M. Dynamic interneuron-principal cell interplay leads to a specific pattern of in vitro ictogenesis. Neurobiol Dis 2018;115:92-100.

https://doi.org/10.1016/j.nbd.2018.04.004.

Ludwig KA, Uram JD, Yang J, Martin DC, Kipke DR. Chronic neural recordings using silicon microelectrode arrays electrochemically deposited with a poly(3,4-ethylenedioxythiophene) (PEDOT) film. J Neural Eng 2006;3:59-70. https://doi.org/10.1088/1741-2560/3/1/007.

McNaughton BL, O'Keefe J, Barnes CA. The stereotrode: A new technique for simultaneous isolation of several single units in the central nervous system from multiple unit records. J Neurosci Methods 1983;8:391-7. https://doi.org/10.1016/0165-0270(83)90097-3.

Misra A, Burke JF, Ramayya AG, Jacobs J, Sperling MR, Moxon KA, et al. Methods for implantation of micro-wire bundles and optimization of single/multi-unit recordings from human mesial temporal lobe. J Neural Eng 2014;11:026013. https://doi.org/10.1088/17412560/11/2/026013. 
Misra A, Long X, Sperling MR, Sharan AD, Moxon KA. Increased neuronal synchrony prepares mesial temporal networks for seizures of neocortical origin. Epilepsia 2018;59:636-649. https://doi.org/10.1111/epi.14007

Mormann F, Kornblith S, Quiroga RQ, Kraskov A, Cerf M, Fried I, et al. Latency and Selectivity of Single Neurons Indicate Hierarchical Processing in the Human Medial Temporal Lobe. J Neurosci 2008;28:8865-72. https://doi.org/10.1523/JNEUROSCI.1640-08.2008.

O'Keefe J, Recce ML. Phase relationship between hippocampal place units and the EEG theta rhythm. Hippocampus 1993;3:317-30. https://doi.org/10.1002/hipo.450030307.

Paik S-J, Park Y, Cho DD. Roughened polysilicon for low impedance microelectrodes in neural probes. J Micromechanics Microengineering 2003;13:373-9. https://doi.org/10.1088/0960$1317 / 13 / 3 / 304$.

Park YS, Hochberg LR, Eskandar EN, Cash SS, Truccolo W. Early detection of human focal seizures based on cortical multiunit activity. 2014 36th Annu Int Conf IEEE Eng Med Biol Soc 2014; 5796-5799. https://doi.org/10.1109/EMBC.2014.6944945.

Podkorytova I, Hoes K, Lega B. Stereo-Encephalography Versus Subdural Electrodes for Seizure Localization. Neurosurg Clin N Am 2016;27:97-109.

https://doi.org/10.1016/j.nec.2015.08.008.

Quiroga RQ, Reddy L, Kreiman G, Koch C, Fried I. Invariant visual representation by single neurons in the human brain. Nature 2005;435:1102-7.

https://doi.org/10.1038/nature03687.

Ranck JB. Studies on Single Neurons in Dorsal Hippocampal Formation and Septum in Unrestrained Rats. Exp Neurol 1973;14:461-555. https://doi.org/10.1016/00144886(73)90290-2; https://doi.org/10.1016/0014-4886(73)90291-4.

Reddy L, Poncet M, Self MW, Peters JC, Douw L, van Dellen E, et al. Learning of anticipatory responses in single neurons of the human medial temporal lobe. Nat Commun 2015;6:8556. https://doi.org/10.1038/ncomms9556v. 
Roehri N, Pizzo F, Lagarde S, Lambert I, Nica A, McGonigal A, et al. High-frequency oscillations are not better biomarkers of epileptogenic tissues than spikes: HFOs Are Not Better Than Spikes. Ann Neurol 2018;83:84-97. https://doi.org/10.1002/ana.25124.

Rutishauser U, Ross IB, Mamelak AN, Schuman EM. Human memory strength is predicted by theta-frequency phase-locking of single neurons. Nature 2010;464:903-7. https://doi.org/10.1038/nature08860.

Rutishauser U, Ye S, Koroma M, Tudusciuc O, Ross IB, Chung JM, et al. Representation of retrieval confidence by single neurons in the human medial temporal lobe. Nat Neurosci 2015;18:1041-50. https://doi.org/10.1038/nn.4041.

Schevon CA, Ng SK, Cappell J, Goodman RR, McKhann G Jr, Waziri A, Branner A, Sosunov A, Schroeder CE, Emerson RG. J Microphysiology of Epileptiform Activity in Human Neocortex. Clin Neurophysiol 2008;25:321-330. https://doi.org/10.1097/WNP.0b013e31818e8010 Schevon CA, Tobochnik S, Eissa T, et al. Multiscale recordings reveal the dynamic spatial structure of human seizures. Neurobiol Dis 2019;127:303-311.

https://doi.org/10.1016/j.nbd.2019.03.015.

Staresina BP, Reber TP, Niediek J, Boström J, Elger CE, Mormann F. Recollection in the human hippocampal-entorhinal cell circuitry. Nat Commun 2019;10:1-11.

https://doi.org/10.1038/s41467-019-09558-3

Stead M, Bower M, Brinkmann BH, Lee K, Marsh WR, Meyer FB, et al. Microseizures and the spatiotemporal scales of human partial epilepsy. Brain. 2010 Sep;133:2789-97. https://doi.org/10.1093/brain/awq190.

Talairach J, Tournoux P, Musolino A, Missir O. Stereotaxic exploration in frontal epilepsy. Adv Neurol 1992;57:651-88.

Truccolo W, Donoghue JA, Hochberg LR, Eskandar EN, Madsen JR, Anderson WS, et al. Singleneuron dynamics in human focal epilepsy. Nat Neurosci 2011;14:635-41.

https://doi.org/10.1038/nn.2782.

Wilson M, McNaughton B. Dynamics of the hippocampal ensemble code for space. Science 1993;261:1055-8. https://doi.org/10.1126/science.8351520. 
Xia M, Wang J, He Y. BrainNet Viewer: a network visualization tool for human brain connectomics. PloS One 2013;8:e68910. https://doi.org/10.1371/journal.pone.0068910.

Yang P-F, Zhang H-J, Pei J-S, Tian J, Lin Q, Mei Z, et al. Intracranial electroencephalography with subdural and/or depth electrodes in children with epilepsy: Techniques, complications, and outcomes. Epilepsy Res 2014;108:1662-70.

Yger P, Spampinato GL, Esposito E, Lefebvre B, Deny S, Gardella C, et al. A spike sorting toolbox for up to thousands of electrodes validated with ground truth recordings in vitro and in vivo. eLife 2018;7:e34518. https://doi.org/10.7554/eLife.34518.

Zijlmans M, Worrell GA, Dümpelmann M, Stieglitz T, Barborica A, Heers M, et al. How to record high-frequency oscillations in epilepsy: A practical guideline. Epilepsia 2017;58:130515. https://doi.org/10.1111/epi.13814. 


\section{SUPPLEMENTARY MATERIAL}

A
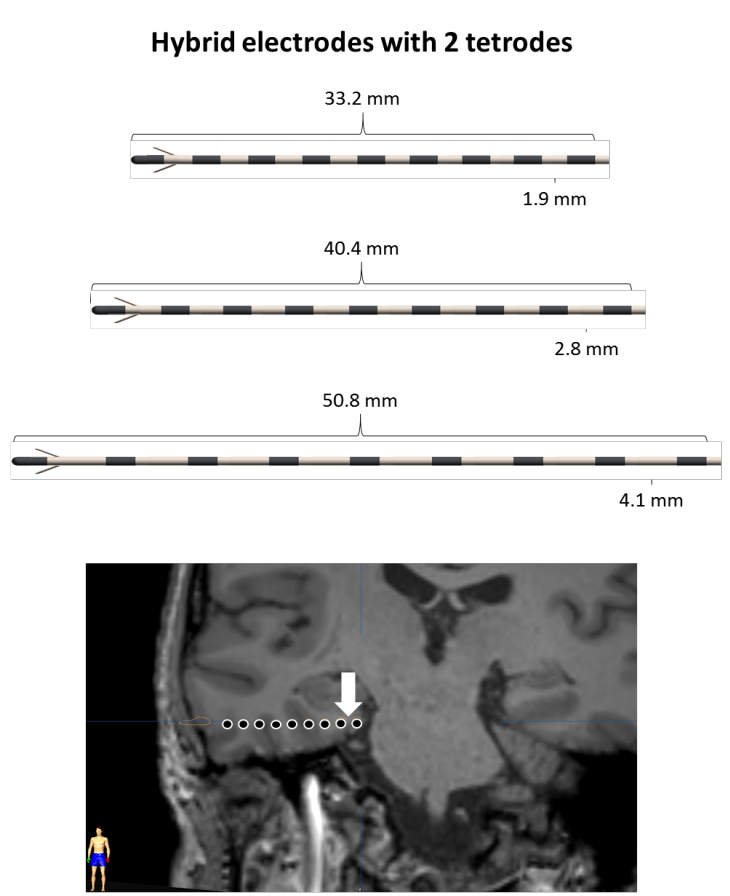

$B$ Hybrid electrodes with 3 tetrodes
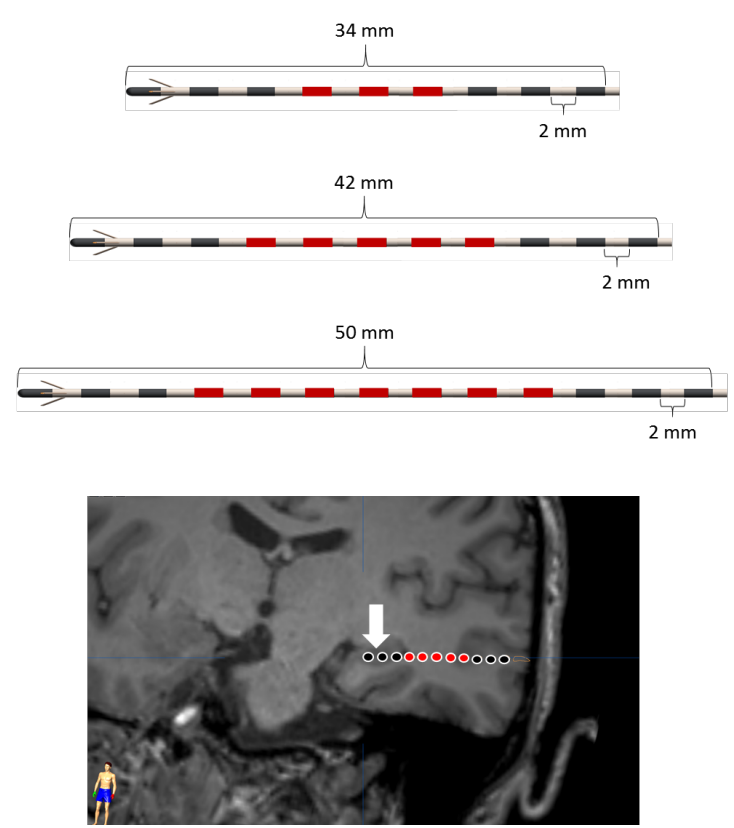

Figure S1 - Illustration of the different models of hybrid electrodes. Three different lengths are available comprising either two or three tetrodes. (A) Models with 2 tetrodes and 8 macrocontacts (black rectangles). The illustration shows a hybrid electrode with two tetrodes, in which the distal macrocontacts are located in the rhinal cortex of a patient. The white arrow indicates the location of the microelectrodes, between the first and the second most medial macrocontacts. (B) Models with 3 tetrodes and 6 active macrocontacts (black rectangles). The contacts in red are inactive. The illustration shows a hybrid electrode with three tetrodes, in which distal macrocontacts are located in the hippocampus of a patient. The white arrow indicates the location of the microelectrodes, between the first and the second most medial macrocontacts. 